\title{
Fault Diagnosis Method of Voltage Sensor in 3-phase AC/DC PWM Converters
}

\author{
Hyung-Seop Kim *,Won-Sang Im *,Jang-Mok Kim *, \\ Dong-Choon Lee **, and Kyo-Beum Lee***
}

\begin{abstract}
This paper proposes a fault diagnosis method of the line-to-line voltage sensors in 3-phase AC/DC pulse width modulation (PWM) converters. The line-to-line voltage sensors are an essential device to obtain the information of the grid voltages for controlling the 3phase AC/DC PWM converters. If the line-to-line voltage sensors are mismeasured by various faults, the voltage sensors can obtain wrong information of the grid voltage. It has an adverse effect on the control of the converter. Therefore, the converter causes the unbalance input $\mathrm{AC}$ current and the DC-link voltage ripple in the 3-phase AC/DC PWM converter. Hence, fast fault detection and fault tolerant control are needed. In this paper, the fault diagnosis method is proposed and verified through simulations and experiments.
\end{abstract}

Keywords: 3-phase PWM converter, Fault diagnosis, voltage sensor

\section{Introduction}

3-phase AC/DC pulse width modulation (PWM) converters have been increasingly employed in recent years due to their advantages such as a unity power factor and a controllable high-quality dc output voltage [1]. The reliability of the converters has been always important issue due to the costs of unplanned stops [2-3]. Hence the converters require a fault immune characteristic which must have fault detection and tolerant control function [4-7]. Specifically, this paper focused on a line-to-line voltage sensor fault among the various faults. When a fault of the line-to-line voltage sensors occurs, a wrong voltage is measured even if the input grid voltage is healthy. Accordingly, the wrong value becomes the input of the phase locked loop (PLL). Therefore, it is impossible to control the converter normally. Hence the converter causes the unbalanced input AC currents and the DC-link voltage ripple. These may result in malfunctions of the PWM converter or cause secondary faults. Accordingly, there is high possibility of secondary fault in the converter system, the load and the grid [8]. Hence, fast fault detection and fault tolerant control are needed. There are many papers about 3-phase AC/DC converter, but there are few papers

\footnotetext{
* Dept. of Electrical Engineering, Pusan National University, Korea. (jmok@pusan.ac.kr)

** Dept. of Electrical Engineering, Yeungnam University, Korea (dclee@yu.ac.kr)

*** Dept. of Electronic Engineering, Ajou University, Korea. (Kyl@ajou.ac.kr)

Received 31 July 2012; Accepted 26 August 2012
}

about the voltage sensor fault of the converter. This paper proposes a fault diagnosis method for the grid-side sensors of the 3-phase AC/DC PWM converter. After the fault detection, the converter can return to normal operation through the proposed method when a fault of a voltage sensor occurs.

\section{Fault diagnosis method of voltage}

\subsection{Influence of fault in voltage sensor}

Fig. 1 shows the block diagram of the 3-phase AC/DC PWM converter with three voltage sensors. It is essential to measure the voltages of the AC input source for controlling the converter. These voltages can be obtained from the voltage sensors. They become the inputs of PLL as shown in Fig. 2. The grid phase-angle $(\theta)$ is acquired through d-q transformation and the PI current controller. Fig. 3 shows the control flow of converter. The voltages $\left(V_{d}^{e}, V_{q}^{e}\right)$ and

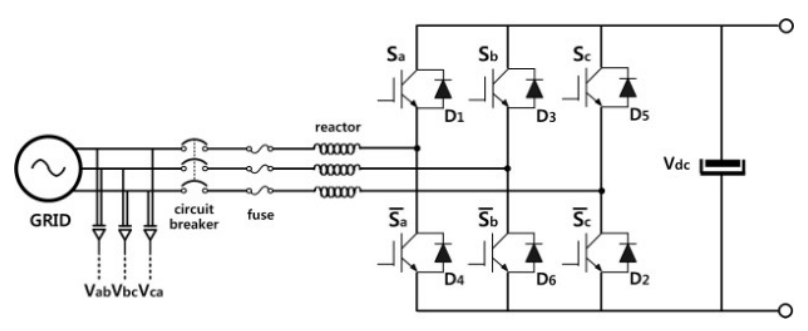

Fig. 1. 3-Phase AC/DC PWM converter structure 


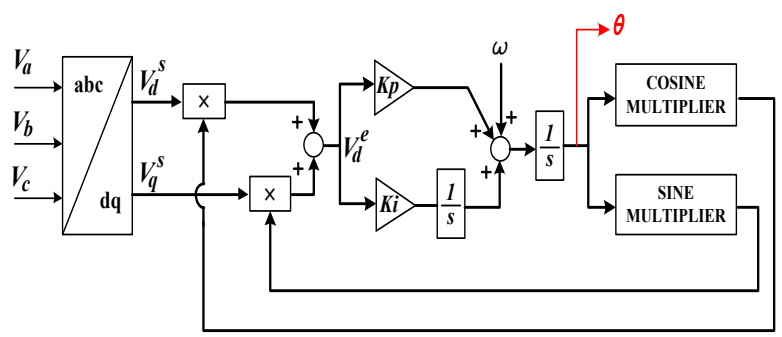

Fig. 2. Structure of PLL

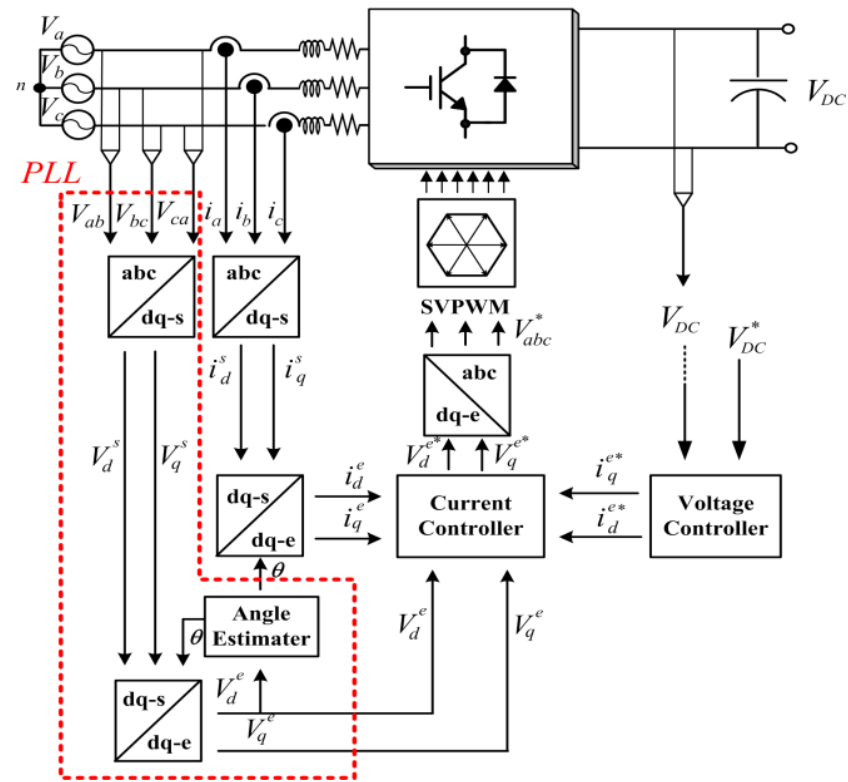

Fig. 3. Control flow of converter

currents $\left(I_{d}^{e}, I_{q}^{e}\right)$ of the synchronous reference frame are calculated by using the grid phase-angle $(\theta)$, and these are the inputs of current controller. The outputs of SVPWM are generated from the reference voltages $\left(V_{a b c}^{*}\right)$ which are the outputs of the current controller. Accordingly, the desired reference DC voltage can be supplied normally in healthy operation condition. However, in the case of a fault occurrence in voltage sensors, the wrong voltage value is obtained. Then the fault voltage enters the input of PLL. As shown in Fig. 3, accordingly the grid phase-angle which is the output of PLL is estimated incorrectly due to the voltage value obtained by the faulted sensor. The wrong grid phaseangle causes the wrong output of $\mathrm{d}-\mathrm{q}$ transformation. Therefore, the inputs $\left(I_{d}^{e}, I_{q}^{e}\right)$, and $\left(V_{d}^{e}, V_{q}^{e}\right)$ of the current controller are distorted, and then distorted output of the current controller generates the six distorted outputs in SVPWM. Thus, the converter becomes in abnormal operation. As a result, the converter has the unbalanced input AC current and the DC-link voltage ripple.

\subsection{Classification of fault in voltage sensor}

The various faults of a voltage sensor can be broadly classified into two types. Specifically the one is a gain-fault and another is a null-fault. The former, a gain-fault, is caused by damages of internal resistors and measurement resistor of the voltage sensor. This gain-fault distorts the amplitude of obtained line-to-line voltage by the voltage sensor. The latter, a null-fault, is caused by the open-circuit inside the voltage sensor. If a null-fault occurs, the measured voltage value is convergent up to ' 0 '. This paper describes the diagnosis method about a null-fault and a gain-fault in the converter.

\subsection{Fault diagnosis of voltage sensor}

Fig.4 shows the flowchart of the proposed fault diagnosis method. In step 1, the line-to-line voltages are measured. In step 2, a sum of voltages is calculated. When voltage sensors have no problem, the sum can be ideally represented as (1).

$$
\begin{gathered}
V_{\text {sum }}=V_{a b}+V_{b c}+V_{c a}=0 \\
\text { where, }\left(\begin{array}{l}
V_{a b}=V_{m} \sin \omega t \\
V_{b c}=V_{m} \sin (\omega t-120) \\
V_{b c}=V_{m} \sin (\omega t+120)
\end{array}\right)
\end{gathered}
$$

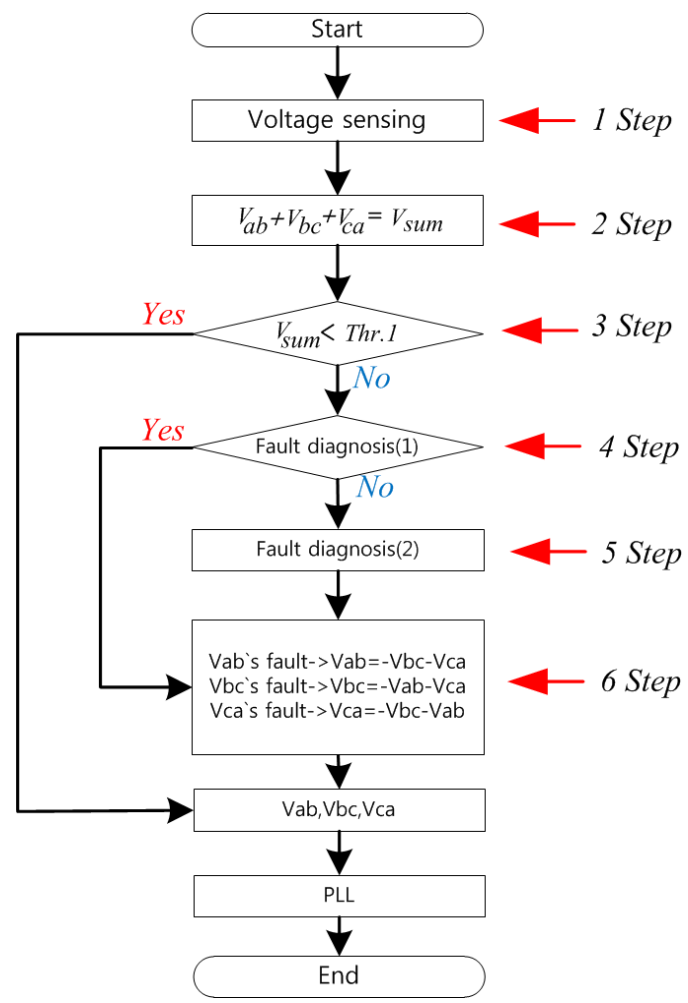

Fig. 4. Flowchart of fault diagnosis method 
However $\mathrm{V}_{\text {sum }}$ is difficult to be zero due to the effect of measurement errors such as offset, scale, quantization errors and non-linearity. Hence, in step 3, (2) is used to find out whether or not a fault of the sensors occurs.

$$
\left|V_{a b}+V_{b c}+V_{c a}\right|<t h r .1
$$

If $\left|\mathrm{V}_{\text {sum }}\right|$ is larger than threshold.1 one of three voltage sensor has a problem at least. In step 4, whether or not a null-fault occurs is determined. At the same time, which voltage sensor occurs the null-fault is decided. In this fault case, the value and the variation of voltage are ideally convergent up to 0 . However, both are difficult to be exactly convergent up to 0 due to forementioned various measurement errors. A null-fault sensor can be detected through comparing the measured voltages and their variations with threshold 2 as (3).

$$
\begin{aligned}
& \left\{\Delta V_{a b} \text { and } \quad V_{a b}\right\}<\text { thr.2 } \\
& \left\{\Delta V_{b c} \text { and } \quad V_{b c}\right\}<\text { thr. } 2 \\
& \left\{\Delta V_{c a} \text { and } \quad V_{c a}\right\}<\text { thr. } 2
\end{aligned}
$$

In step 5, which voltage sensor of three occurs a gainfault is determined. In this case, the sum of 3-phase voltages is not zero but sine wave. If $V_{\text {sum }}$ compares with the faulted voltage, they are inphase or antiphase each other, and the amplitudes of them are different as shown in (4).

$$
\begin{aligned}
& V_{\text {sum }}=V_{a b}+V_{b c}+V_{c a}+Z=Z \\
& \left(Z=k \cdot V_{m} \sin \omega t \quad \text { or } \quad k \cdot V_{m} \sin (\omega t+120) \quad \text { or } \quad k \cdot V_{m} \sin (\omega t-120)\right. \\
& (-1 \leq k \leq 1)
\end{aligned}
$$

Thus, the ratios of two voltage slopes and the ratios of two voltage amplitudes are equal at the same time as (5). Therefore, one of (5) must be true under some fault conditions. After that, which voltage sensor has a gain-fault can be determined by using (5).

$$
\begin{aligned}
& \frac{V_{\text {sum }}}{V_{a b}}=\frac{\Delta V_{\text {sum }}}{\Delta V_{a b}} \\
& \frac{V_{\text {sum }}}{V_{b c}}=\frac{\Delta V_{\text {sum }}}{\Delta V_{b c}} \\
& \frac{V_{\text {sum }}}{V_{c a}}=\frac{\Delta V_{\text {sum }}}{\Delta V_{c a}}
\end{aligned}
$$

In (5), there can be a measurement error of a sensor as above. Because of that, (5) is hard to be true. If one of sensors occurs a gain-fault, it is detected through the comparison with the absolute value and the threshold 3 by (6). In step 6, the wrong measured value can be replaced with the sum of the rest two sensors as (7).

$$
\begin{aligned}
& \left|\frac{V_{s u m}}{V_{a b}}-\frac{\Delta V_{s u m}}{\Delta V_{a b}}\right|<t h r .3 \\
& \left|\frac{V_{s u m}}{V_{b c}}-\frac{\Delta V_{s u m}}{\Delta V_{b c}}\right|<t h r .3 \\
& \left|\frac{V_{s u m}}{V_{c a}}-\frac{\Delta V_{s u m}}{\Delta V_{c a}}\right|<t h r .3 \\
& V_{a b}=-\left(V_{b c}+V_{c a}\right) \\
& V_{b c}=-\left(V_{c a}+V_{a b}\right) \\
& V_{c a}=-\left(V_{a b}+V_{b c}\right)
\end{aligned}
$$

This method can be implemented quickly within only two sampling time because the variations of voltage requires the present value and the previous one as shown in (8).

$$
\begin{aligned}
& \Delta V_{\text {sum }}=V_{\text {sum }}(n)-V_{\text {sum }}(n-1) \\
& \Delta V_{a b}=V_{a b}(n)-V_{a b}(n-1) \\
& \Delta V_{b c}=V_{b c}(n)-V_{b c}(n-1) \\
& \Delta V_{c a}=V_{c a}(n)-V_{c a}(n-1)
\end{aligned}
$$

\section{Simulation}

This section verifies validity of the proposed fault diagnosis method through several simulations. The first example (Ex.1) is that the value of the $V_{a b}$ decreases by

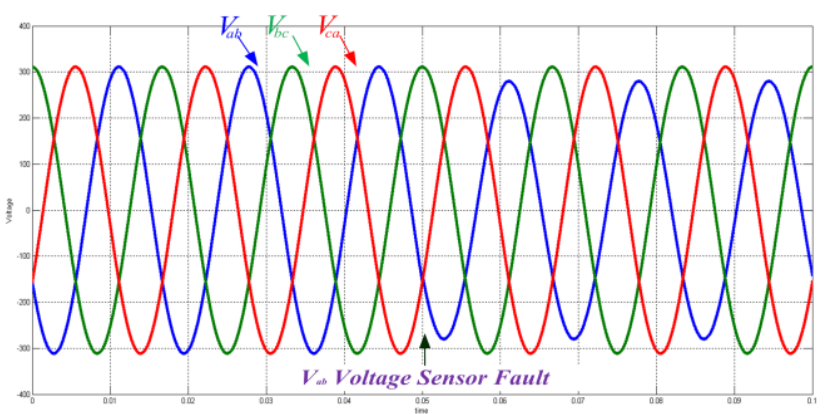

Fig. 5. Three line-to-line voltages (Ex.1) 
(a)

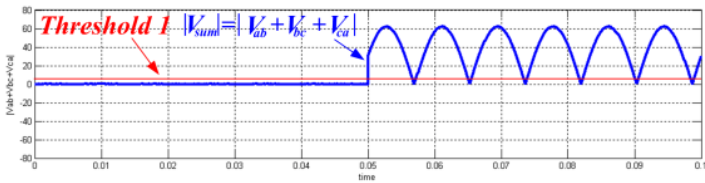

(b)

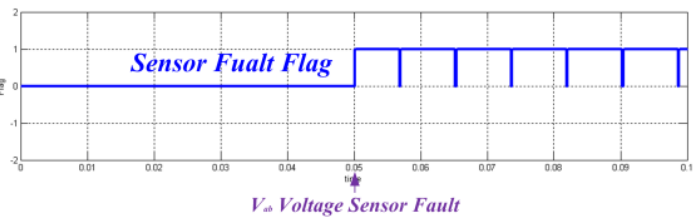

Fig. 6. Sum of three line-to-line voltage and flag signal (Ex.1)

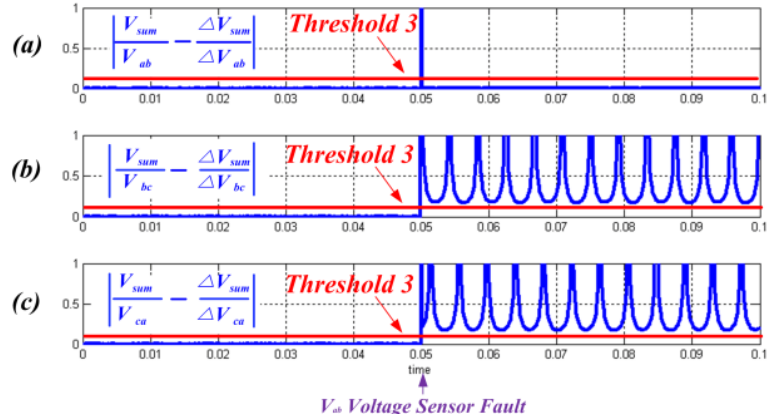

Fig. 7. Simulation results of (6) (Ex.1)

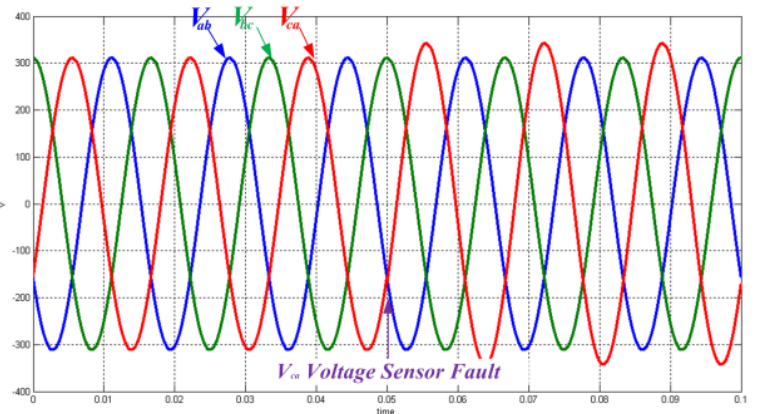

Fig. 8. Three line-to-line voltage (Ex.1)

0.9 times due to a gain-fault of $V_{a b}$ sensor. The results of step 1 are shown in Fig. 5. The sum of line-to-line voltages can be obtained. In step 2, 3, $\left|V_{\text {sum }}\right|$ is compared with threshold 1 as shown in Fig. 6. Because $\left|V_{\text {sum }}\right|$ is larger than threshold 1, a occurrence of fault is decided. In step 4, because $\left|\Delta V_{a b}\right|$ and $\left|V_{a b}\right|$ are upper than threshold 2, a null-fault doesn 't occur. Fig.7(a) shows the results of step 5. Because the value of (6-1) is lower than threshold 3, a gainfault in the sensor of the $V_{a b}$ is regarded as the fault. In the last step 6, the faulted $V_{a b}$ can be replaced with the sum of $V_{b c}$ and $V_{c a}$. For another example(Ex.2), $V_{c a}$ is measured by 1.1 times due to a gain-fault of a sensor as shown in Fig. 8. After a fault, because the results of (6-3) are equal to Fig. 9, a gain-fault of the $V_{c a}$ sensor can be detected. Hence $V_{c a}$ is obtained through the sum of $V_{a b}$ (a)

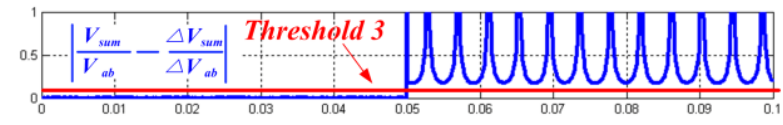

(b)

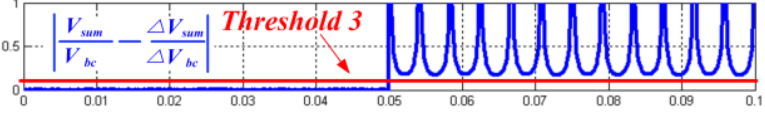

(c)

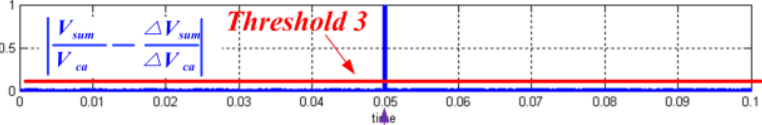

$V_{\text {au }}$ Voltage Sensor Fault

Fig. 9. Results of (6) (Ex.1)

and $V_{b c}$. Therefore, value of fault voltage sensor is replaced with the calculated value by using the two healthy sensors. As a result, the imbalance of the input AC current and the ripple of the output DC voltage disappear.

\section{Experiments}

Experiments are implemented to verify the algorithm of the proposed fault diagnosis method under the same conditions as simulations. As the first example (Ex.1) of simulations, $V_{a b}$ is measured by 0.9 times when a gainfault occurs in the sensor of $V_{a b}$. Fig. 10 shows three lineto-line voltages. As shown in Fig. 11, one of three sensors has a fault in step 3. (3) is unsatisfied as shown in Fig. 12.

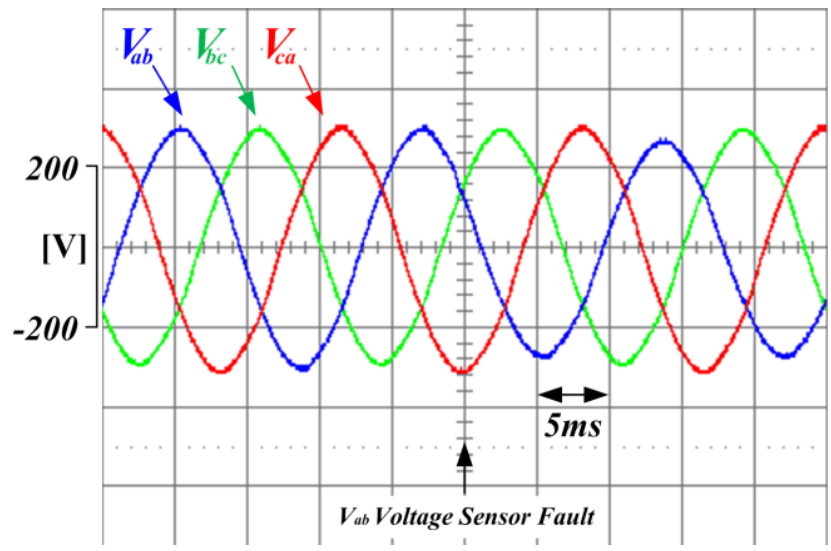

Fig. 10. Three line-to-line voltage (Ex.1)

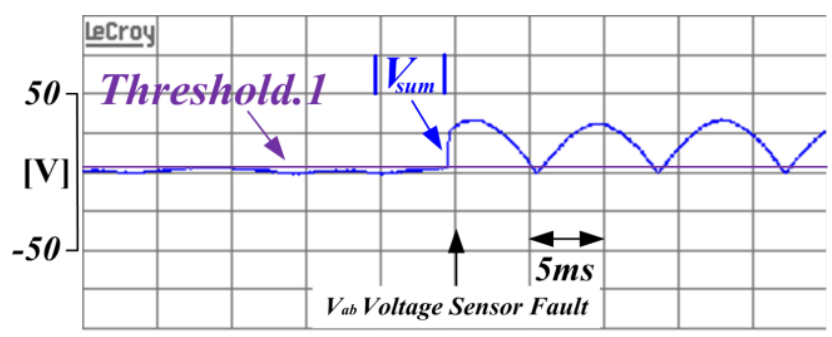

Fig. 11. Sum of three line-to-line voltage (Ex.1) 


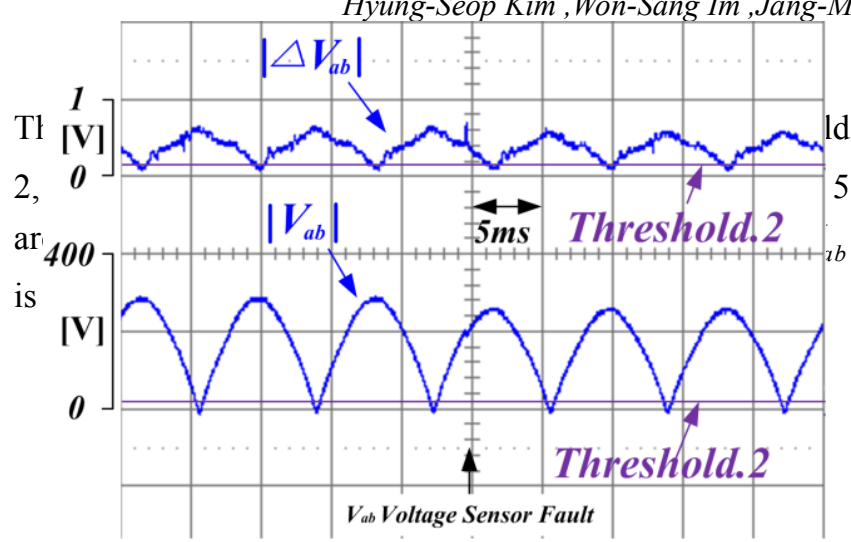

Fig. 12. Experimental results of (3) (Ex.1)

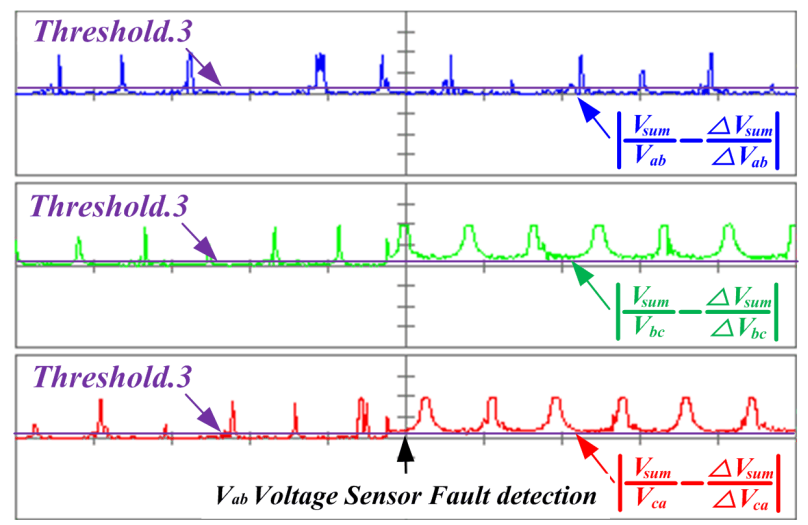

Fig. 13. Experimental results of (6) (Ex.1)

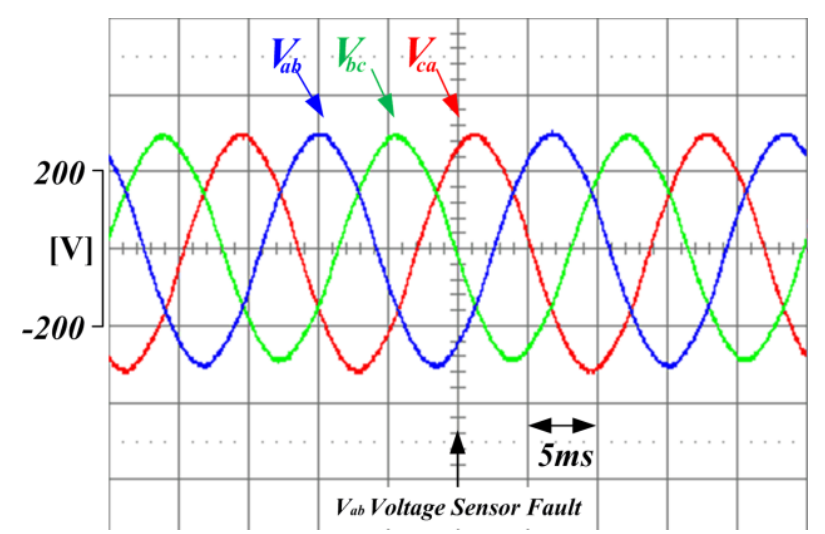

Fig. 14. Three line-to-line voltage (Ex.1)

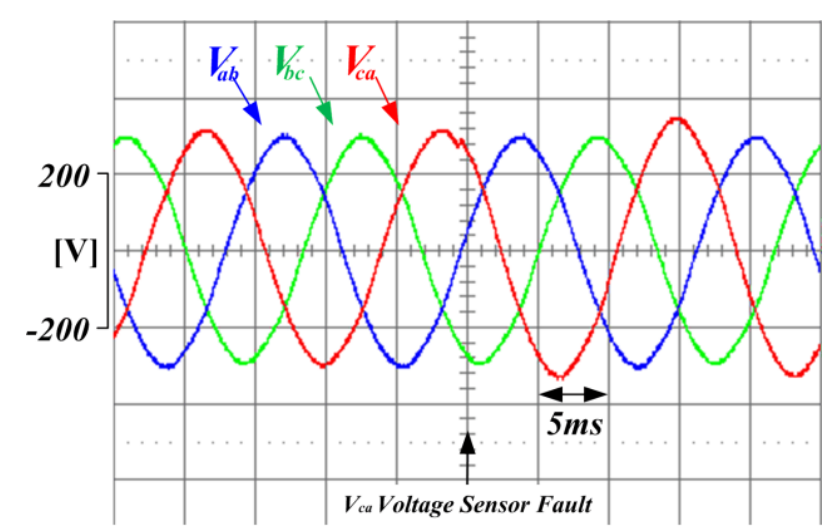

Fig. 15. Three line-to-line voltage (Ex.2)
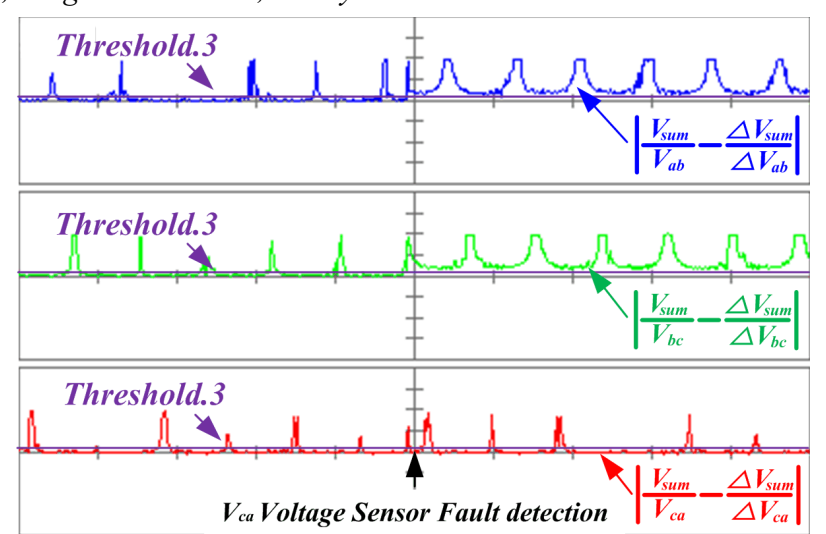

Fig. 16. Experimental results of (6) (Ex.2)

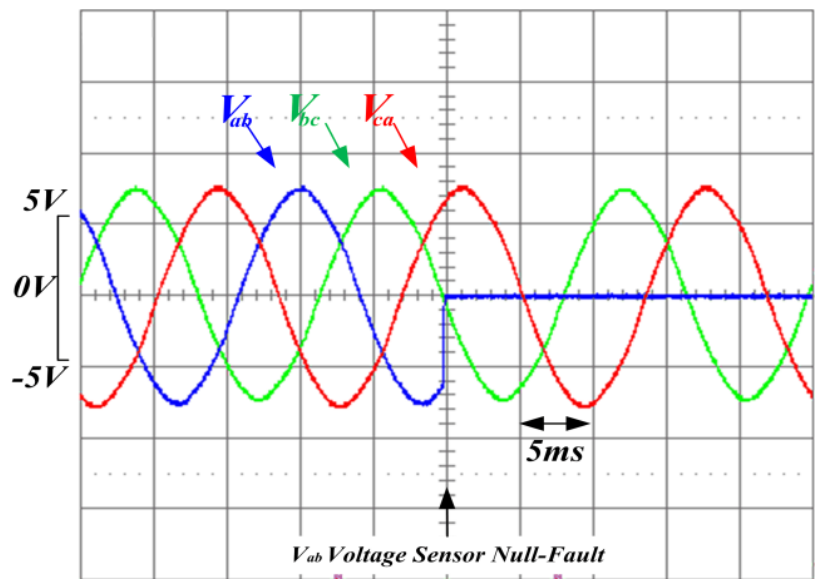

Fig. 17. Three line-to-line voltage (Ex.3)

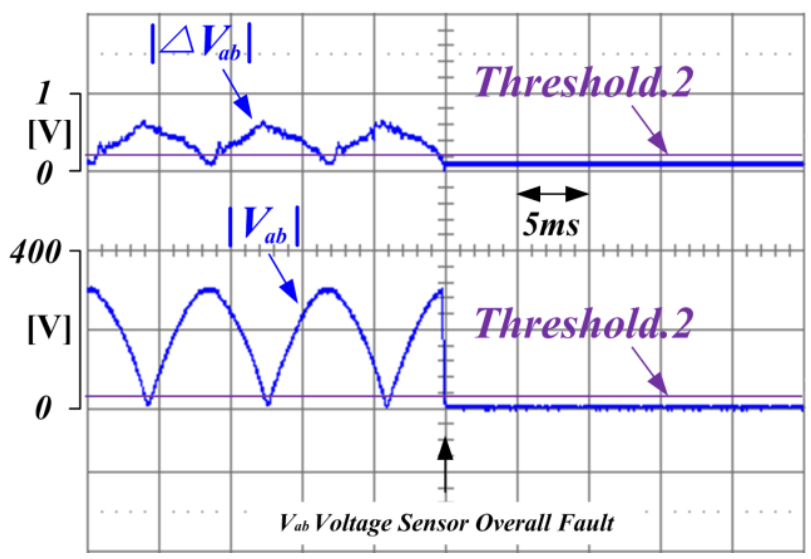

Fig. 18. Experimental results of (3) (Ex.3)

The faulted value of $V_{a b}$ is replaced with the calculated value by using the two healthy sensors (7-1). Next example (Ex.2) is the same as the condition of the second simulation, which the amplitude of $V_{c a}$ increases by 1.1 times. Fig. 15 shows (Ex.2). As shown in Fig. 16, a gain-fault of $V_{c a}$ sensor is confirmed by using (6-3). In the last experiment (Ex.3) as shown in Fig. 17, a sensor of $V_{a b}$ is regarded as a null-fault by using (3-1). Fig. 18 shows the 
results. The validity of the proposed algorithm is verified through three experiments

\section{Conclusion}

This paper analyzes a fault of voltage sensor case and proposes a fault diagnosis method of voltage sensor in 3phase AC/DC PWM converter. The proposed fault tolerant control method can improve the performance and operate normally when a fault of voltage sensor is detected. This method is easily implemented without any additional H/W by using $\mathrm{S} / \mathrm{W}$. The usefulness of the proposed method is verified through simulations and experiments.

\section{Acknowledgements}

This work was supported by KESRI (Korea Electrical Engineering and Science Research Institute) (2009T100100651), which is funded by MKE(Ministry of Knowledge Economy).

\section{References}

[1] Yongsug Suh and Thomas A. Lipo, "Control Scheme in Hybrid Synchronous Stationary Frame for PWM AC/DC Converter Under Generalized Unbalanced Operating Conditions" IEEE Trans. Ind. Appl., vol.42,no3,pp.825835,May/Jun.2006

[2] R. L. de Araujo Ribeiro, C.B. Jacobina, E.R.C. da Silva, and A.M.N. Lima, "Fault detection of open-switch damage inverters using wavelet and SVM," journal of Power Electronics, Vol. 9, No.3, pp. 377-385, Jun. 2009

[3] Young-Jong Ko and Kyo-Beum Lee, "Fault Diagnosis of a Voltage-Fed PWM Inverter for a Three-Parallel Power Conversion System in a Wind Turbine," Journal of Power Electronics, vol. 10, no. 6, pp. 686-693, Nov 2010

[4] M. Beltrao de Rossiter Correa, C. Brandao Jacobian, E.R. Cabral da Silva and A.M Nogueira Lima, "An Induction Motor Drive System with Improved Fault Tolerance" IEEE Trans. Ind. Appl., vol. 37, no. 3, pp.873-879, May/Jun.2001

[5] Yuwen Hu, Lanhong Zhang, Wenix Huang and Feifei Bu, "A Fault-Tolerant Induction Generator System Based on Instantaneous Torque control(ITC)"," IEEE Trans. Energy Conversion, vol. 25, no. 2, pp.412-421,Jun.2010

[6] B.A Welchko, T.A. Lipo, T.M. Jahns and S.E.schulz, "Fault Tolerant Three-Phase AC Motor Drive Topologies: A Comparision of Feature, Cost, and Limitation", IEEE Trans. Power Elec., vol. 19,no. 4,pp.1108-1116, July.2004.

[7] C.B.Jacobian, I.S Freitas, T.M. Oliveria, E.R.C. da Silva, and A.M.N. Lima, "Fault-Tolerant Voltage-Fed PWM Inverter AC Motor Drive Systems", IEEE Trans. Ind. Elec., vol. 51, no. 2, pp. 439-446, Apr.2004.

[8] Won-Sang Im, Jang-Sik Kim, Jang-Mok Kim, Dong-Choon Lee, and Kyo-Beum Lee, "Diagnosis methods for IGBT Open Switch Fault Applied to 3-phase AC/DC PWM
Converter", Journal of Power Electronics, Vol. 12, No. 1,pp.120-127,Jan.,2012.

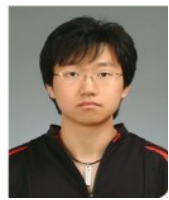

Hyung-Seop Kim was born in Daegu, Korea, in 1985. He received his B.S. in Electrical Engineering from Pusan National University, Busan, Korea, in 2011. Now, He is currently working toward his M.S. degree His current research interests are in the areas of analysis, modeling, design, and control of power converter

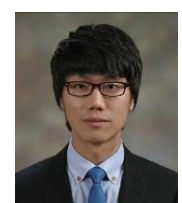

Won-Sang Im was born in Busan, Korea, in 1981. He received his B.S. and M.S. in Electrical Engineering from Pusan National University, Busan, Korea, in 2007 and 2009, respectively. $\mathrm{He}$ is currently working toward his Ph.D. degree at Pusan National University. His research interests include power conversion and electric machine drives.

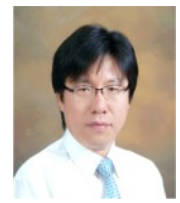

Jang-Mok Kim was born in Busan, Ko rea, in August 1961. He received his B. S. from Pusan National University in 1 988, and his M.S. and Ph.D. from Seou 1 National University, Korea, in 1991 a nd 1996, respectively, in the department of Electrical Engineering. From 1997 to 2000, he was a Senior Research Engineer with the Korea Electrical Pow er Research Institute (KEPRI). Since 2001, he has been with the School of Electrical Engineering, Pusan Nationa 1 University (PNU), where he is currently a Faculty Me mber. In addition, he is a Research Member of the Rese arch Institute of Computer Information and Communicati on at PNU. His present interests include the control of electric machines, electric vehicle propulsion, and power quality.

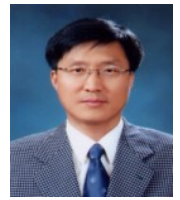

Dong-Choon Lee received his B.S., M.S., and Ph.D. in Electrical Engineering from Seoul National University, Seoul, Korea, in 1985, 1987, and 1993, respectively. He was a Research Engineer with Daewoo Heavy Industry from 1987 to 1988 . Since 1994, he has been a faculty member in the Department of Electrical Engineering, Yeungnam University, Gyeongbuk, Korea. He is serving as a Publication Editor, Journal of Power Electronics, the Korean Institute of Power Electronics, Korea. 
As a Visiting Scholar, he joined the Power Quality Laboratory, Texas A\&M University, College Station in 1998, the Electrical Drive Center, University of Nottingham, U.K. in 2001, and the Wisconsin Electric Machines \& Power

Electronic Consortium, University of Wisconsin, Madison in 2004. Currently, He is joining the FreeDM System Center, University of North Carolina State. His research interests include ac machine drives, control of power converters, wind power generation, and power quality.

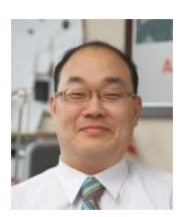

Kyo-Beum Lee was born in Seoul, Korea, in 1972. He received the B.S. and M.S. degrees in Electrical and Electronic Engineering from the Ajou University, Korea, in 1997 and 1999, respectively. He received the Ph.D. degree in Electrical Engineering from Korea University, Korea in 2003. From 2003 to 2006, he was with the Institute of Energy Technology, Aalborg University, Aalborg, Denmark. From 2006 to 2007, he was with the Division of Electronics and Information Engineering, Chonbuk National University, Jeonju, Korea. In 2007 he joined the School of Electrical and Computer Engineering, Ajou University, Suwon, Korea. He is an associated editor of the IEEE Transactions on Power Electronics, IEEE Transactions on Industrial Electronics and the Journal of Power Electronics. He has received two IEEE prize paper awards. His research interests include electric machine drives and renewable power generations. 